\title{
ХИРУРГИЯ ЭКССУДАТИВНЫХ ПЕРИКАРДИТОВ
}

\author{
Д. мед. наук, проф. Лигоненко А. В. \\ Украина, г. Полтава, Украинская медицинская стоматологическая академия
}

DOI: https://doi.org/10.31435/rsglobal_sr/31032019/6382

\begin{abstract}
ARTICLE INFO
Received 16 January 2019

Accepted 16 March 2019

Published 31 March 2019

\section{KEYWORDS}

pericarditis, pericardial effusion, pericardioscopy, thoracoscopy, miniinvasive interventions, surgical treatment.

ABSTRACT

In the article are presented modern approaches to diagnostics, etiological search and methods of surgical correction of pericardial effusion (PE) of different etiology. PE being the most common disease of the pericardium, often has a secondary nature. The overview of modern literature data is presented about terminology and classification of PE, the possibility of etiological search for the cause of effusion in the pericardial cavity, the main diagnostic methods, as well as the main tasks and methods of pericardial surgical interventions and its indications. Based on the analysis of literary sources, we can conclude that the consensus on the clinical and nosological structure, effectiveness and necessity of using various tests and interventions, clinical progression, epidemiology, prognosis and the reasons for the unsatisfactory results of treated PE is actually absent. Our analysis indicates the absence of a systematic and differential approach to choice of treatment tactics.
\end{abstract}

Citation: Лигоненко А. В. (2019) Hirurgiya Ehkssudativnyh Perikarditov. Science Review. 3(20). doi: 10.31435/rsglobal_sr/31032019/6382

Copyright: (C) 2019 Лигоненко А. B. This is an open-access article distributed under the terms of the Creative Commons Attribution License (CC BY). The use, distribution or reproduction in other forums is permitted, provided the original author(s) or licensor are credited and that the original publication in this journal is cited, in accordance with accepted academic practice. No use, distribution or reproduction is permitted which does not comply with these terms.

Введение. Постановка диагноза экссудативного перикардита (ЭП), как правило, не вызывает трудностей, существует множество хирургических вмешательств, предложенных для лечения данной патологии, однако многие авторы продолжают обсуждать мероприятия, направленные на выявление его этиологической причины, преимущества и недостатки различных оперативных вмешательств. Следует отметить существенное количество идиопатических случаев (более $25 \%)$ и рецидивов ЭП (15-60\%), послеоперационных осложнений и высокие показатели послеоперационной летальности (3-42\%) $[15,19,20,23,28,29,34]$. Вопросы выбора хирургического вмешательства, его объема при ЭП различной этиологии являются наиболее актуальными. На сегодняшний день предпочтение отдается методикам, позволяющим минимизировать оперативную травму и уменьшить длительность послеоперационного восстановления. Однако общепринятым является применение какого-либо одного хирургического вмешательства, который зависит не столько от определенного клинического случая, а от возможностей клиники, где это происходит и от выполняющего его специалиста.

Цель нашей работы - изучение и анализ предложенных на сегодняшний день методов диагностики и хирургического лечения больных с экссудативными перикардитами.

Результаты исследования. В связи с ухудшением уровня жизни и различной доступностью медицинской помощи разных слоев населения, усовершенствованием диагностических и лечебных мероприятий, ежегодно количество пациентов с ЭП возрастает [3, 21].

ЭП имеют различную природу возникновения и клиническую картину. Единично встречаются первичные перикардиты, но в большинстве случаев ЭП имеет вторичный характер. Выпот в перикарде развивается на фоне различных заболеваний, усугубляя их течение, прогноз и заставляет вносить коррективы в тактику лечения $[1,2,7,12,21]$. Не всегда удается выявить причину перикардита $[1,2,6,30,25]$. Поиск этиологической причины возникновения выпота в перикарде является основной проблемой в его диагностике и лечении $[2,21,25]$. 
Последние рекомендации Европейской ассоциации кардиологов по диагностике и ведению пациентов с заболеваниями перикарда, опубликованные в 2015 году, не имеют четкой доказательной базы и основаны на выводах отдельных специалистов, лимитированных исследованиях, общепринятых положениях [19]. На сегодняшний день отсутствуют широкомасштабные рандомизированые исследования, изучающий этиологию, распространенность, механизмы и клиническое течение данной патологии.

Среди этиологических факторов развития ЭП наиболее распространенными являются вирусные (30-55\%), бактериальные (3-12\%), системные (20-30\%) заболевания [7, 12, 15, 21, 29$]$. Вследствие травм либо оперативных вмешательств на грудной клетке со вскрытием перикардиальной полости, последующим развитием постперикардиотомного синдрома, также часто наблюдается возникновение ЭП $[11,17]$, также как при наличии инородных тел в полостях грудной клетки (пули, обломки и др.). Данные процессы могут быть инфицированными с развитием септического процесса и накоплением гнойного содержимого [17]. Наличие онкологического процесса [31, 37], химио- и лучевая терапия у пациентов [26, 30, 36] также является достаточно распространенной причиной развития ЭП.

Рецидив накопления жидкости в полости перикарда и/или возникновение констриктивных процессов по данным различных авторов встречается у 15-30\% больных $[19,20,21,23,25,34]$. По данным M. Imazio (2012) [22] и L.S. Lylli (2013) [25] наиболее склонны к возникновению рецидивов аутоиммунные, вирусные и идиопатические перикардиты. Рецидивируют неопластические перикардиты значительно реже при проведении комплексной терапии основного заболевания. Следует отметить, что значимой причиной хронизации и рецидивов ЭП является неполноценное и незавершенное лечение $[6,28,34]$. По данным литературы существует связь данного процесса с распространением воспаления на прилегающую к перикарду соединительную и жировую ткань, эпикард и субэпикардиальный слой, что может способствовать возникновению рецидивов после окончания консервативной терапии $[18,21]$.

Смертность при экссудативных перикардитах зависит от различных факторов, включая этиологию, давность процесса, тяжесть проявлений основного заболевания, своевременность и полноценность оказания медицинской помощи. При вирусных поражениях перикарда смертность составляет 1,1\%, в то время как при гнойных $85-90 \%$ [12, 15, 32].

Острота и давность патологии непосредственно отражается на клинической картине, количестве выпота в перикардиальной полости и скорости его накопления, выраженности основной и сопутствующей патологии. В течение длительного периода жалобы могут отсутствовать при постепенном накоплении и небольшом количестве перикардиального выпота. При быстром накоплении экссудата сердечная недостаточность развивается и прогрессирует быстро и при незначительном объеме жидкости $[5,7,8,12,24]$.

Сердечная и/или дыхательная недостаточность различной степени проявления вызывает основные жалобы пациентов с ЭП: одышку и чувство тяжести за грудиной $[5,7,8]$. Больные часто отмечают повышение температуры тела, отечность ног или лица, слабость, головокружение, кашель $[7,8,12]$. При посттравматических перикардитах клинически чаще значимы характер и степень тяжести непосредственно травматических повреждений $[11,17]$, у пациентов с онкологической патологией - проявления оановного процесса, общей раковой интоксикации [26, 31]. При наличии септического процесса развитие клиники гнойного перикардита может быть невилировано [32].

Тампонада сердца является острым и жизнеугрожающим состоянием при ЭП. Ее развитие связано с критическим нарастанием давления в перикардиальной полости вследствие скопления жидкости, в результате возникает механическое давление на сердце. Р.Р. Сулиманов и Р.А. Сулиманов [9] в своих исследованиях 2013 года показали, что тампонада сердца развивается у $15 \%$ больных идиопатическими перикардитами и $60 \%$ больных неопластическими, туберкулезными и гнойными перикардитами. Тампонада сердца быстро вызывает правожелудочковую или тотальную сердечную недостаточность, что в 8-20\% случаев заканчивается летальным исходом. Только своевременное оперативное вмешательство для эвакуации содержимого из полости перикарда дает возможность спасти жизнь больного [19].

Для ЭП при общем осмотре пациента характерными являются отечность лица и / или нижних конечностей, пульсация-набухание яремных вен шеи, периферический цианоз $[3,4,7$, 24]. При аускультации выявляются приглушенность тонов сердца, тахикардия, различные 
нарушения ритма, шум трения перикарда $[1,4,5,7,16]$. Однако при значительном количестве экссудата тоны сердца могут не выслушиваться вовсе $[3,7,29]$.

Из инструментальных методов диагностики ЭП наиболее часто используют рентгенографию органов грудной клетки, электрокардиографию (ЭКГ), трансторакальную и трансэзофагальную эхокардиоскопию (ЭхоКС), компьютерную (КТ) и магнитно-резонансную томографию (МРТ) [13, 16, 28].

Электрокардиография не показывает специфических нарушений при воспалении перикарда. Наиболее характерно снижение вольтажа комплекса QRST в стандартных отведениях, инверсия зубца $\mathrm{T}$, подъем сегмента ST, иногда возникает тахикардия либо мерцательная аритмия $[7,13,16]$.

Данные рентгенографии свидетельствуют об увеличение размеров сердца и позволяют заподозрить наличие выпота в перикардиальной полости. Характерная трапециевидная форма сердца описывается авторами как «графин с водой». Следует дифференцировать данные изменения от кардиомегалии, новообразований переднего средостения, миокардита и т.д. [7].

Ультразвуковое исследование является наиболее информативным методом диагностики ЭП, которое предоставляет возможность оценить количество жидкости в перикарде $[29,35]$. В норме в полости сердца содержится около 15-35 мл жидкости. Увеличение объема жидкости в полости по данным ЭхоКс представляется в виде сепарации листков перикарда. M.S. Horowitz et al. [35] еще в 1974 году предложили классификацию перикардиальных выпотов, основанную на диастазе листков перикарда по данным ЭхоКС. При диастазе листков перикарда во время диастолы менее 1,0 см объем выпота считается незначительным, а в полости содержится около 100 мл жидкости; при диастазе листков перикарда 1,0-2,0 см - умеренным, при объеме жидкости 100-500 мл; при диастазе более 2,0 см - значительным с содержанием более 500 мл выпота; и очень большим при диастазе более 2,0 мм с наличием признаков тампонады сердца. Данная классификация на сегодняшний день остается актуальной и применяется в клинической практике. При большом количестве выпота в перикарде типичной является картина «качающегося сердца», определяется парадоксальное движение створок митрального и аортального клапана, асинхронное сокращение межжелудочковой перегородки $[7,13]$.

Ультразвуковое исследование позволяет выявить и оценить изменения листков перикарда, наличие посторонних включений [13]. Данный метод позволяет диагностировать развитие сдавления и тампонады сердца. Признаками тампонады сердца являются: зависимость тока крови через митральный и трехстворчатый клапаны от фаз дыхания, отсутствие спадения нижней полой вены на высоте вдоха, коллабирование правых отделов сердца [29]. Простота и доступность ЭхоКС позволяет выполнять ее многократно с целью оценки тяжести, динамики патологического процесса, развития осложнений, эффективности лечения.

Только в $25 \%$ случаев при неинвазивном обследовании определяются этиологическая причина ЭП по данным исследования Румбешта В.В. и соавт. (2008). Специфичность применения инвазивных методов диагностики в сочетании с лабораторными исследованиями составляет $92 \%$ [2].

Самым распространенным вмешательством при лечении ЭП является перикардиоцентез c целью удаления избыточной жидкости. Манипуляция позволяет достаточно быстро нивелировать сдавление и тампонаду сердца. [3, 21, 31]. Европейская ассоциация кардиологов рекомендует при наличии тампонады сердца, большого количества жидкости, рецидивирующего ЭП (уровень доказательности С, класс 1) проведение диагностического перикардиоцентеза. Доступы по Морфану или Ларрею применяются наиболее часто. Рентгенологический или ЭхоКС контроль и ЭКГ мониторинг снижают риски травмирования сердца $[15,28,31,33,36]$.

Сакович В.А. и соавт. (2005г.) [10] используют перикардиоцентез в качествет первого этапа хирургического лечения ЭП для уменьшения степени сдавливания сердца с последующим более радикальным хирургическим вмешательством. Некоторые авторы утверждают, что при отсутствии возможности выполнения радикальных вмешательств, чрезкожный перикардиоцентез может быть рекомендован исключительно при угрозе тампонады сердца $[9,15,36]$.

На сегодняшний день перикардиотомия с перикардиоскопией считается наиболее специфическим и чувствительным методом. При сравнении эффективности перикардиоцентеза 
с частичной перикардэктомией на основании анализа результатов лечения 102 пациентов с ЭП, чрезкожная пункция перикарда показала большее количество осложнений $(61,9 \%$ против 5,1\%), меньшую диагностическую ценность $(28,6 \%$ против $71,4-79,5 \%)$, и требовала более длительного пребывания пациентов в условиях стационара (22,1 день против 8,3 дней соответственно) по данным исследования Сулиманова Р.Р. (2013.) [9]

Многие авторы утверждают, что информативность биопсии перикарда и эпикарда, цитологического исследования выпота ниже у пациентов с острым течением ЭП, чем у больных с хроническим и рецидивирующим течением заболевания $[18,33]$.

Признаки сдавливания сердца, развитие и прогрессирование гемодинамических нарушений, неэффективность консервативного лечения, хронизация и рецидив процесса, развитие констрикции сердца являются показанием к оперативному лечению [3, 19, 25, 28, 37].

Предложено много разновидностей вмешательств для лечения ЭП. Как правило, используют полную или частичную срединную стернотомию, переднюю миниторакотомию, субксифоидальный или торакоскопических доступ или переднебоковую торакотомию [30,37]. В большинстве случаев выполняют частичную или субтотальную перикардэктомии, создание сообщения с плевральной или брюшной полостями $[10,33]$.

Миниинвазивные методики являются наиболее распространенными в лечении ЭП на сегодняшний день. Внеплевральная субксифоидальная и черезплевральная перикардиотомии в различных вариациях являются самыми популярными среди них $[9,10,28,38,39]$. Исследователи M. Liberman et al. (2005) сравнивали результаты хирургического лечения с выполнением торакоскопического и субксифоидального доступа для формирования фенистрации перикарда. Среди пациентов, которых оперировали с применением торакоскопических вмешательств смертность была значительно ниже, чем среди тех, кто перенес субксифоидальную перикардиотомию (0,16\% против 34,6\%) [38].

H.A. Gumrukuoglu et al. (2011) продемонстрировали результаты лечения 100 пациентов с выпотными перикардитами. Пациенты были разделены на три группы: у I группы пациентов использовали чрезкожную пункцию и дренирование перикарда, у II группы - первичное миниинвазивное вмешательство, у III группы - перикардиоцентез с последующим оперативным вмешательством. Рецидив заболевания значительно чаще возникал в I группе больных $(26 \%$ против 8 и $11 \%$ во II и III группах). Показатели ранней и поздней летальности были самыми низкими во II группе (3\% и 8\% соответственно), в I группе показатели были несколько выше (5\% и $8 \%$ соответственно), в III результаты были достоверно выше, чем в остальных (7\% и 15\% соответственно) [28].

Широкая торакоскопическая перикардэктомия считается методом выбора у больных с онкологической этиологией перикардитов по мнению Н. Uramoto и T. Hanagiri (2010) [39]. S. Celik et al. (2012) [37] в своих исследованиях обращают внимание на безопасность и эффективность левосторонней миниторакотомии у пациентов с онкологической патологией не зависимо от наличия или отсутствия тампонады сердца.

Доля субтотальных перикардэктомий, выполняемых через стернотомный и торакотомные доступы, значительно уменьшилась с развитием миниинвазивных вмешательств. Только в случае констриктивных изменений, неэффективности ранее выполненных вмешательств стоит прибегать к более радикальным вмешательствам [28].

Остаются достаточно высокими цифры послеоперационной летальности при лечении пациентов с ЭП. По данным различных авторов они достигают 30-40\% при гнойных перикардитах и остаются на уровне 2,3-3,6\% при вирусных, неосложненных негнойных ЭП [15, 28, 29].

\section{Выводы:}

- на сегодняшний день остаются не до конца решенными вопросы клиниконозологической структуры, эпидемиологии, течения, прогноза ЭП и причин неудовлетворительных результатов их лечения;

- неинвазивные методы исследования почти неинформативны в диагностике этиологических причин ЭП, однако позволяют выявить наличие и тяжесть данной патологии;

- на сегодня отсутствует единое мнение касательно целесообразности, эффективности и необходимости разных исследований в диагностике ЭП. Мы считаем, что это связано, в первую очередь, с различными методами анализа, неоднородностью и ограниченностью исследуемого материала. 
- анализ отечественных и иностранных исследований, посвященных наиболее распространенным методам оперативного лечения ЭП, позволяет утверждать, что не существует дифференциального, обоснованного подхода к выбору тактики ведения данной группы пациентов, учитывающего состояние больного, наличие сопутствующей патологии.

\section{ЛИТЕРАТУРА}

1. Арутюнов Г. П. Перикардит. Современные проблемы диагностики и лечения / Г. П. Арутюнов // Сердце. - 2006. - № 8. - С. 384-400.

2. Возможности дифференциальной диагностики перикардитов неясного происхождения / В. В. Румбешт, А. А. Дюжиков, А. И. Кислицкий [и др.] // Kardiol Serdečno-Sosud Hir. - 2008. - № 6. - С. 64-66.

3. Гирялевский С. Р. Диагностика и лечение заболеваний перикарда: современные подходы, основанные на доказательной информации и клиническом опыте / С. Р. Гирялевский. - М.: Медиа Сфера, 2004. - 132 с.: ил.

4. Заболевания перикарда: учебно-методическое пособие / М. М. Михно, А. М. Пристром, Т. Д. Тябут [и др.]. - Минск: [б. и.], 2011. - 49с.

5. Иванов К. М. Клинико-гемодинамические особенности хронической сердечной недостаточности при поражениях перикарда // Материалы конференции специалистов по сердечной недостаточности, Москва, 2001. - С. 52.

6. О новых звеньях патогенеза хронического перикардита / В. В. Кочмашева, Е. Д. Рождественская, Е. С. Беликов [и др.] // Рациональная фармакотерапия в кардиологии. - 2009. - № 2. - С. $49-54$.

7. Сугак А. Б. Перикардиты: этиология, классификация, клиника, диагностика, лечение. Часть 1 / А. Б. Сугак // Вопросы современной педиатрии. - 2009. -Том 8, № 2. - С. 77-84.

8. Сугак А. Б. Перикардиты: этиология, классификация, клиника, диагностика, лечение. Часть 2 / А. Б. Сугак, Е. И. Алексеева // Вопросы современной педиатрии. - 2009. -Том 8, № 3. - С. 76-81.

9. Сулиманов Р. Р. Сравнительный анализ малоинвазивных хирургических методов лечения выпотного перикардита / Р. Р. Сулиманов, Р. А. Сулиманов // Вестник Новгородского государственного университета. - 2013. - № 71, т. 1. - С. 47-50.

10. Торакоскопическая фенестрация перикарда при экссудативных перикардитах различной этиологии / В. А. Сакович, Д. Б. Дробот, А. В. Аидин [и др.] // Бюллетень ВСНЦ СО РАМН. - 2005. - № 3(41). - С. 86-87.

11. Хирургия повреждений сердца при раневой политравме и шоке / П. Н. Замятин, Я. К. Голобородько, В. В. Бойко, В. В. Булагаю - Харьков: Консум, 2003. - 156 с.: ил.

12. Acute pericarditis / L. E. Tingle, D. Molina, C. W. Calvert // Am Fam Physic. - 2007. - Vol. 76, № 10. P. 1509-1514.

13. Alraies M. C. Should we still use electrocardiography to diagnose pericardial disease? / M. C. Alraies, A. L. Klein // Cleveland Clinic Journal of Medicine. - 2013. - Vol. 80, № 2. - P. 97-100.

14. Consecutive 1127 therapeutic echocardiographically guided pericardiocenteses: clinical profile, practice patterns, and outcomes spanning 21 years / Tsang T.S., Enriquez-Sarano M., Freeman W.K. [et al.] // Mayo Clin Proc. - 2002. - Vol. 77, № 5. - P. 429-436.

15. Controversial issues in the management of pericardial diseases / M. Imazio, D. H. Spodick, A. Brucato [et al.] // Circulation. - 2010. - Vol. 121. - P. 916-928.

16. Could the heart rate play a role in pericardial inflammation? / Z. Khoueiry, C. Roubille, N. Nagot [et al.] // Medical Hypotheses. - 2012. - Vol. 79. - P. 512-515.

17. Delayed pericardial effusion following stab wounds to the chest / D. G. Harris, J. T. Janson, J. V. Wyk [et al.] // Eur J Cardio-Thor Surg. - 2003. - Vol. 23. - P. 473-476.

18. Diagnostic value of pericardial biopsy: improvement with extensive sampling enabled pericardioscopy / P. M. Seterovic, A. D. Ristic, R. Maksimovic [et al.] // Circulation. - 2003. - Vol. 107, № 7. - P. 978-983.

19. ESC Guidelines for the diagnosis and management of pericardial disease / Y. Adler, P. Charron, M. Imazio [et al.] // Eur. Heart J. - 2015. - Vol. 36. - P. 2921-2964.

20. Idiopathic recurrent acute pericarditis: a cross talk between autoimmunity and autoinflammation / D. Rigante, S. Napodano, O. M. Lucherini [et al.] // J Genet Syndr Gene Ther. - 2013. - Vol. 3. - P. 1-6. DOI: 10.4172/2157-7412.S3-003.

21. Imazio M. Management of pericardial effusion / M. Imazio, Y. Adler // Eur. Heart J. - 2013. - Vol. 34. P. 1186-1197.

22. Imazio M. Treatment of recurrent pericarditis / M. Imazio // Expert. Rev. Cardiovasc. Ther. - 2012. Vol. 10. - P. 1165-1172.

23. Laparoscopic pericardectomy for post-cardiac surgery recurrent pericardial effusion / P. Agarwal, R. K. Bagdi, N. Solomon // Bangl J Endosurg. - 2013. - Vol. 1, №2. - P. 20-22.

24. LeWinter M. M. Acute pericarditis / M. M. LeWinter // N Engl J Med. - 2014. - Vol. 371, № 25. P. 2410-2416. 
25. Lilly L. S. Treatment of acute and recurrent idiopathic pericarditis / L. S. Lilly // Circulation. - 2013. Vol. 127. - P. 1723-1726.

26. Malignant cardiac tamponade from non-small cell lung cancer: case series from the era of molecular targeted therapy / B. T. Li, A. Pearson, N. Pavlakis [et al] // J Clin Med. - 2015. - Vol. 4. - P. 75-84.

27. Management of cardiac tamponade: a comperative study between echo-guided pericardiocentesis and surgery - a report of 100 patients / H. A. Gumrukcuoglu, D. Odabasi, S. Akdag, H. Ekim // Cardiol Res Pract. - 2011. - Vol. 2011. - P. 1-7. DOI: 10.4061/2011/197838.

28. Nishimura R. A. Constrictive pericarditis in the modern era: a diagnosis dilemma / R. A. Nishimura // Heart. - 2001. - Vol. 86. - P. 619-623.

29. Pericardial disease: diagnosis and management / M. H. Khandaker, R. E. Espinosa, R. A. Nishimura [et al.] // Mayo Clin Proc. - 2010. - Vol. 85, № 6. - P. 572-593.

30. Pericardiectomy for radiation-induced constrictive pericarditis / N. Barbetakis, T. Xenikakis, D. Paliouras [et al.] // Hell J Cardiol. - 2010. - Vol. 51. - P. 214-218.

31. Pericardiocentesis with cisplatin for malignant pericardial effusion and tamponade / T. Oida, K. Mimatsu, H. Kano [et al.] // World Journal of Gastroenterology. - 2010. - Vol. 16, № 6. - P. 740-744.

32. Purulent pericarditis / L. F. Santos, D. Moreira, P. Ribeiro [et al.] // Rev Port Cardiol. - 2013. - Vol. 32, № 9. - P. 721-727.

33. Purulent pericardial effusions with pericardial tamponade - diagnosis and treatment issues / C. P. Petcu, R. Dilof, C. Bătăios, P. D. Pectu // Cur Health Scien J. - 2013. - Vol. 39, № 1. - P. 53-56.

34. Risk of constrictive pericarditis after acute pericarditis / M. Imazio, A. Brucato, S. Maestroni // Circulation. - 2011. - Vol. 124. - P. 1270-1275.

35. Sensitivity and specificity of echocardiographic diagnosis of pericardial effusion / M.S. Horowitz, C.S. Schultz, E.B. Stinson [et al.] // Circulation. - 1974. - Vol. 50. - P. 239-247.

36. Subxiphoid pericardiostomy in the management of pericardial effusions: case series analysis of 368 patients / N. Bacit, Y. Ünlü, M. Ceviz [et al.] // Heart. - 2005. - Vol. 91. - P. 785-790.

37. Surgical properties and survival of a pericardial window via left minithoracotomy for benign and malignant pericardial tamponade in cancer patients / S. Celik, M. Celik, B. Aydemir [et al.] // World J Surg Oncol. 2012. - Vol. 10, № 123. - P. 1-8. DOI: 10.1186/1477-7819-10-123.

38. Ten-year experience with nontraumatic pericardial effusion: a comparison between the subxyphoid and transthoracic approaches to pericardial window / M. Liberman, C. Labos, J. S. Friedberg [et al.] // Arch Surg. - 2005. - Vol. 140. - P. 191-195.

39. Uramoto H. Video-assisted thoracoscopic pericardiectomy for malignant pericardial effusion / H. Uramoto, T. Hanagiri // Anticancer research. - 2010. - Vol. 30. - P. 4691-4694. 